\title{
Information Exploration Using The Pond
}

\author{
Olov Ståhl, Anders Wallberg, Jonas \\ Söderberg, Jan Humble, Lennart E. Fahlén \\ and Adrian Bullock \\ Swedish Institute of Computer Science (SICS) \\ Box 1263, SE-164 29, Kista, Sweden \\ +4686331500 \\ \{olovs, andersw, jas, humble, lef, \\ adrian\}@sics.se
}

\author{
Jenny Lundberg \\ Department of Human Work Science and Media \\ Technology \\ Blekinge Institute of Technology \\ Box 520, 37225 Ronneby, Sweden \\ +46457385563 \\ Jenny.Lundberg@bth.se
}

\begin{abstract}
In this paper we describe The Pond, a system used to search for and visualise data elements on an engaging tabletop display. The Pond uses methods of unencumbered interaction and audio feedback to allow users to investigate data elements, and supports shoulder-to-shoulder collaboration with the physical Pond artefact mediating the collaboration between those people gathered around it. The user interface is based on an ecosystem metaphor, presenting data elements in the form of shoals of aquatic creatures inside a virtual 3D pond. The Pond is an interactive system offering an appealing and novel way to search for and interchange information. We describe the motivation and design choices behind The Pond, the system as it stands today, details of its implementation, and observations from a study of The Pond in use.
\end{abstract}

\section{Categories and Subject Descriptors}

H.3.3 [Information Storage and Retrieval]: Information Search and Retrieval - Clustering, Information filtering, Search process. H.5.1 [Information Interfaces and Presentation (e.g. HCI)]: Multimedia Information Systems - Artificial, augmented and virtual realities, Audio output, Evaluation/methodology. H.5.2 [Information Interfaces and Presentation (e.g. HCI)]: User Interfaces - Graphical user interfaces (GUI), Prototyping. H.5.3 [Information Interfaces and Presentation (e.g. HCI)]: Group and Organization Interfaces - Collaborative computing, Synchronous interaction, Theory and models.

\section{General Terms}

Design, Experimentation, Human Factors.

\section{Keywords}

Database, visualization, searching, virtual environment.

\footnotetext{
Permission to make digital or hard copies of all or part of this work for personal or classroom use is granted without fee provided that copies are not made or distributed for profit or commercial advantage and that copies bear this notice and the full citation on the first page. To copy otherwise, or republish, to post on servers or to redistribute to lists, requires prior specific permission and/or a fee.

CVE '02, September 30-October 2, 2002, Bonn, Germany.

Copyright 2000 ACM 1-58113-489-4/02/0009...\$5.00.
}

\section{INTRODUCTION}

The constantly increasing amount of information and media available in electronic form leads to a growing demand for new methods for searching and browsing. Traditional text based database queries can be limiting, requiring a user to know exactly what it is she is looking for, and rely on the use of a standard computer interface, typically that of a mouse and keyboard. Furthermore, most computer applications written today are single user applications. Systems that support group collaboration are traditionally run on multiple workstations communicating across a network. In The Pond design we have moved away from the conventional desktop metaphor and established notions of how information systems should be accessed and manipulated. Instead we have made an attempt to build a tool that is easy and intuitive to manage, and exciting and beautiful to experience. The Pond is a desk-based display system that can be used by several users to search for data elements in some information set, e.g., on the Web. It presents the search results and allows user interactions via a graphical and aural user interface. One intention with The Pond is to support co-operation on equal terms around a single display. The Pond is designed to be more fun and social in its use than precise and analytical.

\section{MOTIVATION}

The work on The Pond draws heavily on two earlier locally developed systems, and concepts and experiences can be traced back to these systems. The Web Planetarium, a three-dimensional interpretation of HTML structures in the form of a graph [18], and the Library Demonstrator, an information landscape constructed from the contents of an online library database [8], were both interfaces to active online information. Common features of these systems were the spatial arrangement of data elements, navigation around these data elements and the introduction of new data elements into the display.

While these systems were successful in presenting information to end users, they were not necessarily easy to use. When using the Web Planetarium new information was only introduced as a result of explicit interaction (clicking) by the user and novice users could be too shy to discover this fact and not load new information. The positioning of this new data could also be problematic. In the Planetarium the user must be observant otherwise she may miss the introduction of the new data element. The Library addressed this problem using animation and selforganizing models to show the emerging relationships between 
information, and it is this approach we build upon in The Pond with its shoal metaphor. Navigation around the data used a point and click method, automatically transporting the user to (or close to) the selected object. The core of this navigation technique was to adopt an object centric philosophy where users were explicitly freed from the overhead of having to manage their navigation and movement in three dimensions. This restriction of the overall freedom of movement meant that users were able to focus on the exploration of the information space. However, users still had problems navigating the structures. It could range from getting completely lost to not being able to look upon the data in the way they wanted. These systems were also subject to single user interactions and did not encourage a social atmosphere for the exchange of gathered information.

When we started the work on The Pond, the objective was to design a multimedia user interface where users without any prior knowledge or acquired competence should be able to easily handle both single as well as groups of objects in an affordable and easy way. The objects in turn should be able to represent any type of information or media. They should be able to present themselves in a natural fashion at the users convenience. Also, they should disappear in non-obtrusive fashion when no longer needed. The user should be able to select, move, sort and explore objects of interest without having to confront the rigid hierarchy that is the hallmark of traditional file handling and database applications. Instead of using colour, form or position to indicate group or class membership we wanted to use motion dynamics of objects to indicate these properties. If new information is requested, objects should just float to the surface. Objects no longer relevant should slowly sink to the bottom and quietly disappear. The viewpoint stays fixed above the pond surface. In this way we would achieve a function that did not require the users themselves to navigate.

An observation made early in the design process was the difference in the behaviour of users when confronted with a horizontally placed display vis-à-vis a vertically placed one. When a group of people gathers in front of a vertically placed display be it a big screen TV or a wall projection - immediately a somewhat authorial situation tends to develop. The person in control of the display content is perceived as a teacher or lecturer. The rest of the group will play the role of an audience or school class. When, on the other hand, the display or projection area is positioned horizontally people will gather on a more equal basis. With inspiration from as disparate sources as roulette tables, billiard tables and the military's "classical" tactical war gaming board it was decided that The Pond should use a horizontal display and that none of the sides of the physical artefact should be more important than any of the others.

\section{RELATED WORK}

Tabletop displays have been in use for a number of years now, for example in visualizing information [16], for command and control scenarios [11] and augmenting physical objects [15]. The table provides a natural centre for interaction to take place around and encourages collaboration between the users while they are interacting with the table [2]. The developments in plasma display technology coupled with touch sensitive surfaces now make it possible to dispense with potentially clumsy projected displays in favour of a neat, compact display. Interaction with The Pond is object based, using physical tags to load information into the application by placing them around the edge of the table [14,22]. Unlike other approaches where the physical objects are placed directly on the table and manipulated $[21,9,19]$, the tags used in The Pond are passive. They make it possible to load and store information, but do not manipulate this information any further. Interacting with the contents of The Pond is supported through direct manipulation of the virtual Pond objects [20] using a touch screen display. Typically, interactions with the tabletop systems discussed above make use of stereoscopic glasses, data gloves, and magnetic position trackers. These techniques are not used in The Pond as our aim was to create as easy and direct an interface as possible and not to encumber the user with devices needed to experience the material presented.

Approaches to improve access to online information and to visualise it in an intuitive manner have been under development for a long time. Examples of systems that display information graphically in three dimensions include VR-VIBE [1], QPIT [6] and BEAD [5]. All of these systems require (at least to some extent) the users to use navigation to access particular objects, because objects may obscure each other or may be out of view. However, navigation within 3D spaces is known to be difficult [13], especially if the navigation needs to be precise. The Pond design makes use of a static view of the information space, making viewpoint navigation unnecessary.

\section{THE POND}

We now describe The Pond system in detail, examining its construction, both in terms of the physical artefact and the underlying software, and the way in which it is used.

\subsection{The Pond ecosystem metaphor}

The Pond uses a user interface based on an ecosystem metaphor. The objective was to present an aesthetic that would hide the work chore aspect and act as a complementary backdrop for dialogue. When interacting with The Pond, the users see a 3D presentation of a virtual pool or pond, an aquatic environment in which the information objects resulting from queries are presented as shoals of marine creatures. The visual presentation is complemented by a sound environment, consisting of a number of bubbling, splashing and whirling sounds, indicating various active processes inside the pond virtual environment.

Each information creature inside the virtual pond has a simple navigational behaviour. For instance, creatures belonging to the same shoal, and thus query, attempt to group together, while at the same time avoiding creatures from other shoals. These types of simple rules make the virtual pond a dynamic environment, where shoals of information creatures move around in tight groups, avoiding each other as well as the pond walls, and reacting to interactions from the users.

The Pond does automatic garbage collection, which means that one or several of the shoals that exists in the virtual pond environment may be removed. This may happen for instance if the pond is too crowded or because a particular shoal has not been interacted with for a long time. A shoal that is selected for removal by the system will sink down towards the bottom of the virtual pond, where it will disappear. However, should a user interact with a sinking shoal, e.g., select one of its creatures, the 
shoal will return to the surface and another shoal might be removed instead.

\subsection{The Pond example application}

To investigate the concepts behind The Pond we developed an application that allows users to search for and browse music content on the Internet. The music theme was chosen to maximise The Pond's visual and sonic impact, being a theme common to most potential end-users. However, The Pond is not limited to this domain and is adaptable to any other database resource.

A search task is initiated by a user providing a keyword string, for instance the name of an artist, group, or perhaps a musical genre. This keyword must exist on a preconfigured RFID tag (the creation of arbitrary queries is not currently supported at the user level). The Pond "forwards" the keyword to an Internet search engine and presents the resulting album hits as a shoal of creatures, each representing one specific album. The virtual creatures are represented by simple geometric shapes, which are texture mapped with the albums' cover images. By interacting with a creature, users are able to access the album data (e.g., title) and hear samples from the tracks. The virtual pond itself consists of a 3D model of a deep, narrow shaft that extends down from a watery surface.

The music oriented Pond application was selected since we wanted an application that would allow us to explore and evaluate the ideas behind The Pond without having to implement a lot of advanced search functionality, and which would also be interesting and enjoyable to use (e.g., looking for and listening to your favourite music). Furthermore, the size of a "typical" query result was expected to be less than 50 items, which would allow us to present a number of simultaneous shoals in the virtual pond environment without making it appear too crowded.

\subsection{The hardware platform}

The physical Pond artefact has the form of a large desk, on top of which a large touch-sensitive plasma display is horizontally placed. On top of the display surface is a wide wooden frame with an irregular curved outline, which represents the bank of the virtual water pond rendered on the display. The frame is covered by pieces of thick carpet so that users standing around it can comfortably lean over the display when interacting with the virtual pond environment (Figure 1).

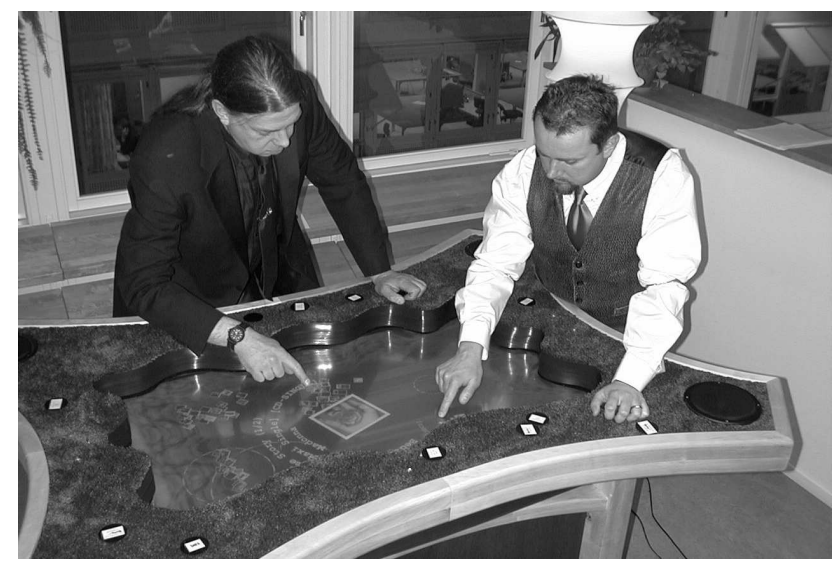

Figure 1: The Pond desk
Built into The Pond frame are a number of speakers that are used to output various sounds and music samples. The use of audio is an important feature in interacting with The Pond, and the sound system consists of several devices including a sampler, subwoofer and amplifier. The Pond frame also encloses several RFID tag readers. Each reader is entirely embedded into the frame carpet and uses three light-emitting diodes to indicate its position and state to The Pond users. Users may initiate queries by placing a RFID tag on such a reader. The tag's identifier, sensed by the reader, will identify a query keyword or phrase and the query will be initiated. The RFID tags can be seen as a hardcoded subset of queries.

\subsection{The software platform}

The Pond software platform consists of three different components:

A visualiser that renders the view from the virtual pond environment on the plasma display.

A Java program (the query application) that interacts with web servers, sending queries and analysing results in the form of web pages.

A Java program (the pond application) that accepts query keywords from the users and communicates these to the query application. This program also uses the results from the query application to introduce and control shoals of creatures in the virtual pond.

The visualiser and the pond application are built using the DivE [10] distributed VR system from SICS. The virtual pond environment is in fact a DIVE virtual world, shared by the visualiser and the pond application. The pond and query applications communicate using a TCP socket connection, through which the pond application sends query keywords from the users (via tag readers), and receives query results in the form of music record information (record titles, artists, links to music samples etc.). Such information is used to create and animate DIVE objects representing creatures in the virtual pond environment. The pond application also handles the audio output (music samples, sound effects, etc.) to The Pond table speakers (see Figure 2).

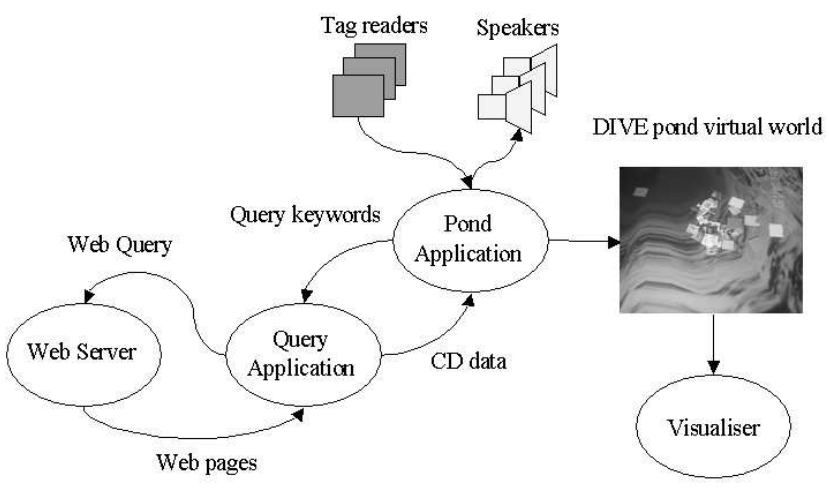

Figure 2: Overview of The Pond software components

\section{INTERACTIONS}

Users standing around The Pond table are able to interact with it in several ways to perform various tasks. In the absence of keyboard or mouse devices, the users perform most interactions 
by tapping or stroking the touch-sensitive display surface. Furthermore, prepared tags that are spread out on top of The Pond frame allow users to input information about queries without having to type on a keyboard.

The conscious actions that may be performed are the following:

$\square \quad$ Initiating a new query

$\square \quad$ Accessing information about query results in the form of shoals of pond creatures

$\square \quad$ Zooming down on a shoal to get a closer look at the creatures

$\square \quad$ Building a selection of creatures from various shoals

$\square \quad$ Saving a selection for later use

$\square \quad$ Uploading a saved selection into the pond

These actions will be described in detail in the following sections.

\subsection{Initiating queries}

Queries are initiated by placing tags on the tag readers in The Pond table frame. When a tag is placed on a reader, the reader senses the tag's unique identifier, which is pre-mapped to a query keyword or phrase. As soon as a query is initiated, an empty shoal appears inside the pond, representing the ongoing query. The shoal is indicated by a circle and a text string specifying the query keyword, as seen in Figure 3.

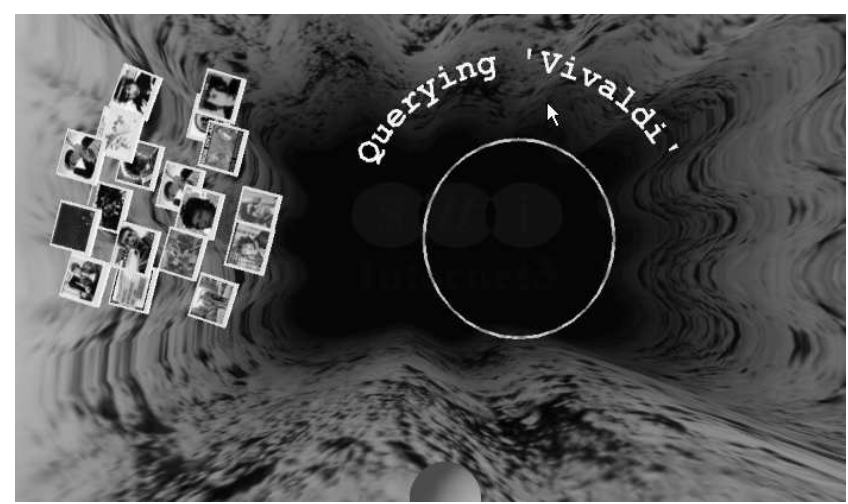

Figure 3: To the right is an empty shoal, representing an ongoing query using the keyword 'Vivaldi'. To the left is a shoal of creatures representing a finished query.

The circle will commence to "float" inside the pond environment, bouncing off the pond walls and avoiding other shoals.

When the query results are initially delivered, creatures start to appear inside the empty query shoal. Each such creature represents an information element from the query result; in this case a CD album. As soon as a particular result creature has been created, it will begin to move around. However, since all creatures resulting from a particular query stay close together, different query shoals are easily identifiable, even with a vastly populated pond environment.

When all the results have been delivered and the corresponding virtual creatures created, the shoal circle label will change to only display the query keyword (see Figure 4). Shortly thereafter the shoal circle and label will disappear, leaving behind only the creatures visible to the users. However, it is possible for users to make the circle and label visible again, something we describe in the next section.

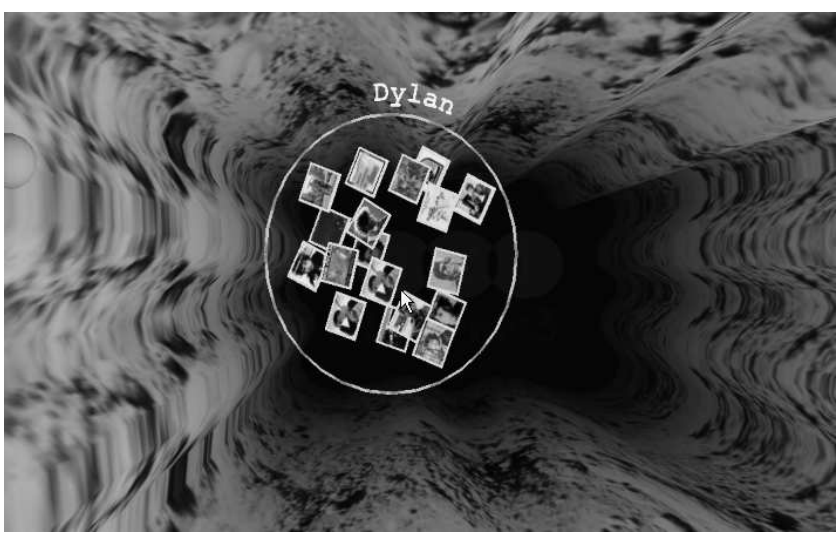

Figure 4: A shoal representing the results of a query using the keyword "Dylan". The shoal circle and keyword text are only visible for a couple of seconds.

\subsection{Accessing query results}

Each shoal member represents an information element that is part of the result of the corresponding query. Users are able to access this information by manipulating the creatures in different ways.

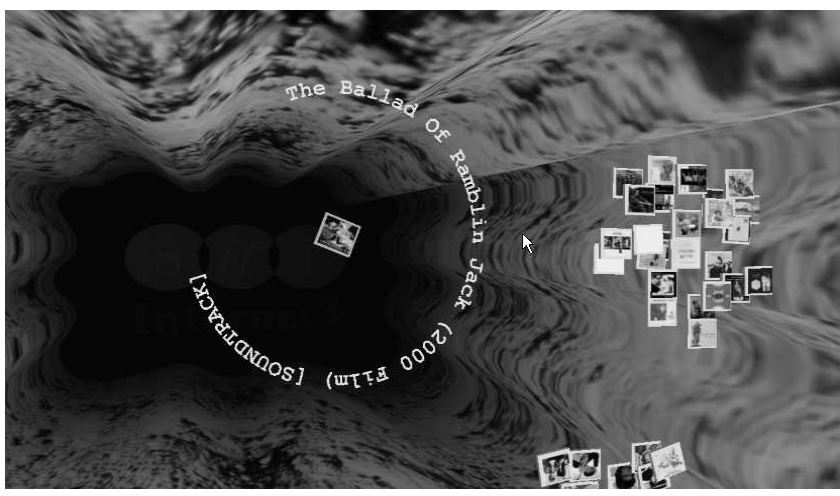

Figure 5: A selected creature

A user selects a creature by tapping on it on the display, and is then able to see the information identifying the artist and title of the corresponding $\mathrm{CD}$. This information is presented as a virtual text string, encircling the 3D-creature and moving alongside it (see Figure 5). The text will only be visible for a brief period (around five seconds) and will then disappear. The frame of the creature will become green to indicate the selection, and will remain so until changed back to white again when another creature is selected.

Tapping once more on an already selected creature activates playback of audio samples from the CD. Sample data is streamed over the network; the creature will then float up to the surface, presenting itself in more detail. (Figure 6). 


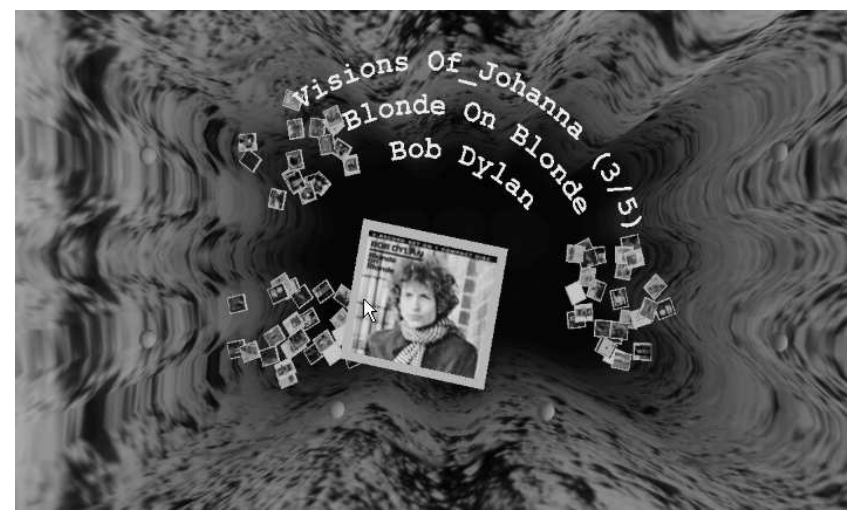

Figure 6: Playing sample three (of five possible) from the CD "Blonde on Blonde"

\subsection{Zooming}

By default, the viewpoint in the virtual pond environment is placed above The Pond, looking down, and at a distance from where the view is always guaranteed to include all the creatures currently inside the pond. In this way the users are able to get a good overview of all the activity within the environment. However, this also constrains the creature representations to be rather small (as seen on the plasma display), which might present a problem when trying to identify the creature graphics of the $\mathrm{CD}$ albums they represent.

In order to allow users to get a closer view of one of the shoals, a zooming mechanism allows for the translation of the virtual viewpoint to a position close to a shoal centre. The view port is just large enough to encompass the whole shoal with the benefit of making the creatures in that shoal together with their associated text strings and images, appear larger. As a result, other shoals may end up out of view, not visible from the new viewpoint position. Another feature of the zooming mechanism is that, while zoomed, the viewpoint is attached to the shoal, which means that it will move as the creatures within the shoal moves. In this way the viewpoint will always stay centred on the chosen shoal, even as this shoal changes its position within the virtual pond. While zoomed, the shoal creatures may be interacted with in the same way as before, e.g., selected to initiate replay of the music samples.

Placing a query tag on a reader, whose corresponding shoal already exists in the pond environment, triggers the zooming mechanism. Removing the tag from the reader will reset the viewpoint to the default overview position. Thus, if a user places a tag associated with the keyword "Dylan" on a reader while a "Dylan" shoal exists in the pond, the viewpoint will change to a position close to this shoal, and stay there as long as the tag is on the reader. Whenever the tag is removed, the viewpoint is reset.

Only one user at a time can use the zoom mechanism. If a zoom is active while a user initiates another zoom the viewpoint state won't be overridden and the second request is ignored.

\subsection{Building a selection}

As users initiate more and more queries, older shoals may have to be removed in order to incorporate newer ones. To prevent a particular creature from being removed from the pond environment, it is possible for users to move individual creatures to safe areas, called creels. This is done by touching the creature with the finger, and then dragging the finger (and thus the creature) along the surface and releasing it over the creel area. Creels exist in several places in The Pond environment, close to the tag readers. Once inside a creel, a creature is constrained to move only within the creel boundaries. By moving several creatures, possibly from different shoals, into a creel, a selection shoal is formed consisting of creatures that a user finds interesting for some reason. Since this particular action shows an interest from the user in a specific data element, this instantiates an extra functionality. A more focused query is automatically launched pertaining to that particular record (usually resulting in fewer hits and consequently a smaller shoal), thus further populating the environment. The creatures in the creels may be interacted with in the same way as other creatures (e.g., tapped on to play music samples), the only difference being that they won't be removed from The Pond environment as long as they stay inside the creel. Creatures that are dragged out of a creel will return to their native shoal, or form a new shoal if their native shoal no longer exists.

\subsection{Saving and uploading a selection}

By using a recordable tag, it's possible for a user to save the contents (i.e., creatures) of a creel selection shoal. A recordable tag is a RFID tag which is not pre-mapped to a search keyword (see "Initiating Queries" earlier). Placing such a tag on a tag reader next to a creel creates an association between the tag and the creatures within the creel area. When the tag is then removed from the reader, the creel's selection shoal will disappear from The Pond environment and may be regarded as being stored on the tag. By placing the same tag on a reader at a later stage, the creatures of the "saved" selection will reappear, added to the corresponding creel shoal. Thus, creels and recordable tags allow users to save references to specific albums, which may be accessed on a later occasion or shared with others.

\subsection{The Pond audio environment}

The sonic environment of The Pond consists of two different parts, the "soundscape" and the interface sounds. In accordance with The Pond ecosystem metaphor, the nature of the soundscape is founded on a family of aquatic whirling sounds and a deep, obscure mumbling giving the impression of the data elements ascending from an abyss of ooze or mud. These ambient sounds fade out when samples from a selected creature starts to play, and fades smoothly in when the music stops. The interface sounds acts as a feedback mechanism to indicate user interactions like selecting/unselecting, clicking, dragging etc. This collection also originates from a number of concrete water sounds. Samples from different types of bubbles, a dripping tap, whirlpools etc. are heavily processed to suit their particular purposes. Examples include:

When a user initiates a query, the appearance of the query shoal is accompanied by the sound of sluggish bubbles rising from the bottom.

When a sound file is retrieved over the network, the waiting time is masked with a bubble vortex that after a few seconds is smoothly merged with the music sample. 
$\square$ When the user removes a RFID tag from a tag reader, the action is accompanied by sounds of a cluster of bubbles being rapidly inhaled by The Pond itself.

When the user draws his finger across the touch screen to drag an object, a glass organ sound reminiscent of drawing a finger along the damp edge of a crystal wineglass is heard.

The visual zooming in of a shoal is illustrated by the familiar bubble sound gradually "magnified" through lowering of the pitch. When zooming out, the process is taken backwards.

Every time an interface sound needs to be played, The Pond system will randomly choose a sound from a collection of sounds available for the particular interaction. There are ten query sounds, ten RFID sounds, ten dragging sounds, etc. The idea is to give the impression of the sonic interface being somewhat organic and unpredictable.

The precise spatial placement of every sound is achieved through a built-in high quality 4-channel sound system. A subwoofer in the table foot produces a deep and suggestive bass. The computer controlled software mixer makes it possible to physically move sounds around and to create expressive musical gestures.

\section{OBSERVATIONS FROM USE}

To test out The Pond a number of sessions were held with external participants in the expectation that some qualitative assessment of the effectiveness of the Pond could be made. In all six sessions were held with a total of nine users, each session lasting for around an hour. A video recording was also made. In addition to the test subjects one of the Pond developers participated in each session, initially presenting The Pond but staying close by in case of questions or problems. Our participants were mostly familiar with computer use, though not familiar with alternative interfaces like The Pond. The focus of the studies was on the technology-in-use [4], i.e., the sequences of interaction within which The Pond came to be used in real time. The emphasis is placed not on "the user", nor on usability strategies as conceived in HCI, but on the "lived work" that users and developers engage in together, in order to make the technology work in situ [7].

\subsection{Encountering The Pond}

In encountering The Pond (Figure 7), users had little difficulty in seeing the sense of The Pond - see its potential utility - as a result of the practical course of instruction into its use. In other words, The Pond became a readily intelligible artifact. Users engaged in various activities, together and alone, such as selecting CDs, listening to samples, transferring CDs to RFID tags, and transferring CDs from RFID tags.

Not only did the users see the sense of The Pond but they also recognized the relevance of The Pond to naturally organized activities of playing music. People are ordinarily constrained by the physicality of musical formats (CDs, audio-tapes, vinyl, etc.), which individualize music and control access to it. The Pond, on the other hand, affords in principle quite a radical alternative: users can select music from a large data-base holding tens of thousands of CDs; they can interrogate and select items of interest to them, they can load music onto portable devices, and they can add new music to The Pond, for example.
As one user explained it:

"Place it in a Virgin Megastore, it can be used as a jukebox. Perhaps you should specialize it more so it fits into a category like film, books, articles, and radio. And you should be able to tell The Pond which CD you want and then be able to put it into your shopping basket."

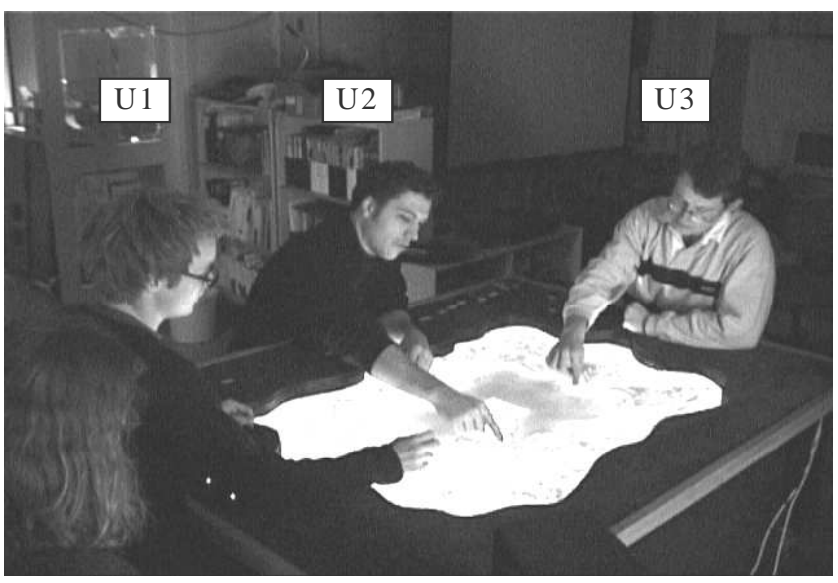

Figure 7:Three users exploring The Pond during one of the evaluation sessions.

\subsection{Interacting with The Pond}

In some sequences of interaction it is noticeable that the sound of The Pond worked as functional feedback. After the users had recognized the connection between the function and the sound, the sound worked as a support for the user in interacting with The Pond. This can be seen in a sequence when a user experienced difficulties in dragging a $C D$ out from her own creel. In the sequence the sound that is heard as her finger passed the sound border of the creel made it clear to her if she had succeeded or not.

The Pond is engineered to support hands-on experience and ease of use, i.e. mainly its touch surface and object oriented presentation of the information displayed. Its simplicity of use is one of its key usability features as seen and understood from a user perspective:

"The main good thing about The Pond is that the interface doesn't require any special level of computer skills for using it." (User of The Pond)

Comments such as these indicate that not only did the users see the sense and recognize the relevance of The Pond, but that they sought to appropriate The Pond. By that we mean that the users could envision its future use for practical purposes in their daily lives, changes withstanding. These changes are crucial to The Pond's appropriation and eventual implementation in practice.

\subsection{Shortcoming of The Pond}

Our studies identified a number of problems with The Pond, areas that future development work should concentrate on.

The ability to manipulate objects requires improvement. Currently users experience some practical difficulty in dragging and turning objects on the interface. In other words, the touch screen needs to be more sensitive and responsive to user actions. 
$\square \quad$ One of the major challenges users identified was the need to be able to search the Pond in a dynamic fashion. For example, it is currently not possible for users to issue custom search queries.

Related to the need to perform active searches is the issue of extending the services offered by The Pond. Thus, as the quote from the user above suggests, there is need to add and develop other services related to music and entertainment.

A multi-interface version of the touch screen is required, enabling users to work individually. The development of multiple-user touch screens should solve this.

Having found objects of personal relevance, appropriation relies on the implementation of multiple means of taking things out of The Pond. This poses the challenge of adapting or devising mobile devices.

The issues emerging from the user studies provide concrete input into an ongoing Cooperative Design process [3]. Conducted with an eye towards an iterative prototyping life-cycle, analytic attention has been paid to the sense and relevance of The Pond as understood from a use perspective, and the practical troubles and tangible future possibilities that impact upon appropriation of The Pond in practice [17].

More general observations, not drawn explicitly from the recorded sessions, showed that the main drawbacks of The Pond are its limited interface flexibility for more elaborate queries and singleuser touch screen interaction. However, once The Pond is populated with reasonable query data its strength of design of concept comes through. The technology did not become obtrusive, and could function as an appealing backdrop and overview when not directly interacted with.

The present day Pond artefact does not provide any other means of input other than pre-designated tag keyword searches (identified above). We aimed towards non-intrusive forms of interaction and avoided introducing keyboard type input. The RFID tags do serve well in cases where a hierarchical, finite and discrete database structure exists, as the music database in our case. Notice that The Pond is in the most part populated by relations and not exact matches, so we force exploration on the users. It proved at times frustrating for users not to be able to directly summon the artist or track of their choice, and at times gratifying in discovering alternative music of their liking.

\section{SUMMARY AND FUTURE WORK}

We have presented The Pond, a multi-user system for browsing information (currently CD record data) on the Web using an engaging tabletop display. Users input musical search keywords (typically names of artists or groups) using RFID tags and are presented with matching Web information in the form of shoals of aquatic creatures inside a virtual $3 \mathrm{D}$ pond. The virtual pond environment is presented on a big touch-sensitive plasma display, which is placed horizontally to better support shoulder-toshoulder collaboration between those people gathered around it. By touching the surface of the display users can interact with the creatures to access the information they represent, e.g. play music samples from the corresponding CDs.

A series of users studies have provided initial insights into the utility of The Pond. The results indicate that the device and metaphor are easy to understand and use, but also identify a number of problem areas. For instance, the touch sensitive display doesn't currently support multiple simultaneous interactions, which sometimes caused the users' actions to interfere with each other.

The Pond has been demonstrated to members of the research community as well as to the public on numerous occasions. The feedback and observations from these sessions have been used to refine The Pond under an ongoing evolution process. Future ideas include support for multiple simultaneous interactions on the display surface, using a voice input system for entering search keywords, and using PDAs to extract and input information to and from The Pond environment.

\section{ACKNOWLEDGMENTS}

This work was undertaken as part of the Swedish Research Institute for Information Technology (SITI) funded Electronic Landscapes Project (ELP) in the Internet 3 research program. We would like to thank all those who have experienced The Pond and provided us with invaluable feedback.

\section{REFERENCES}

[1] Benford, S. D., Snowdon, D. N, Greenhalgh, C. M., Ingram, R. J., Knox, I. and Brown, C. C., "VR-VIBE: A Virtual Environment for Co-operative Information Retrieval", in Proc. Eurographics '95, Maastricht, The Netherlands, 349360, September, 1995

[2] Benford S., et. al., "Designing Storytelling Technologies to Encourage Collaboration Between Young Children", in $\mathrm{CHI}$ 2000, Hague, Netherlands, April 2000, pp.556-563, ACM Press

[3] Bødker, S. and Grønbæk, K., "Design in Action: From Prototyping by Demonstration to Cooperative Prototypin", Design at Work: Cooperative Design of Computer Systems (eds. Greenbaum, J, and Kyng, M.), p. 197-218, Hillsdale, New Jersey: Lawrence Erlbaum Associates, 1991.

[4] Button, G., "The Curious Case of the Vanishing Technology", Technology in Working Order: Studies of Work, Interaction, and Technology (ed. Button, G.), p. 1028, London: Routledge, 1992.

[5] Chalmers M., "Using A Landscape Metaphor to Represent a Corpus of Documents", Proc. European Conference on Spatial Information Theory, Elba, September 1993, pp. 377 390.

[6] Colebourne, A., Mariani, J. A., and Rodden, T., "Q-PIT: a populated information terrain, visual data exploration and analysis III:" in Grinstein, G. G. and Erbacher, R. F. (eds.), SPIE Proc. Ser., 1996, 12-22.

[7] Crabtree, A., Pettifer, S., and West, A., "Inventing New Technologies: The Economics of Information and Situated Evaluation", eSCAPE Deliverable 4.0 Towards a Common Methodology, Esprit Long Term Research Project 25377, p. 85-106, Lancaster University: Computing Department. ISBN 1862200785.1999. 
[8] eSCAPE Deliverable 4.1., "The Library Abstract eSCAPE Demonstrator" (eds. Mariani, J. \& Rodden, T.), Esprit Long Term Research Project 25377, ISBN 186220079 3. 1999

[9] M. Fjeld, et. al., (1999): "Exploring Brick-Based Navigation and Composition in an Augmented Reality", in H.-W. Gellersen (ed.) Handheld and Ubiquitous Computing (HUC 99), Vol 1707, pp.102-116.

[10] Frécon E., Smith G., Steed A., Stenius M. and Stahl O., “An Overview of the COVEN Platform", In Presence:

Teleoperators and Virtual Environments, Volume 10, Issue 1, February 2001, Pages 109 - 127

[11] Penny Chase et. Al., "Semantic and Content Visualization", Coling-Acl 98 workshop: Content Visualization and Intermedia Representations, Montreal, Canada, August 1998.

[12] Hughes, J.A., Crabtree, A., and Rodden, T. (eds.), eSCAPE Deliverable 4.0 "Towards a Common Methodology", Esprit Long Term Research Project 25377, Lancaster University: Computing Department. ISBN 186220078 5. 1999.

[13] Ingram, R., and Benford S., "Legibility Enhancement for Information Visualisation”, in IEEE Visualization '95, Georgia, Atlanta, 1995.

[14]H. Ishii and B. Ullmer, "Tangible Bits: Towards Seamless Interfaces between People, Bits, and Atoms", Proc. ACM CHI'97, pp. 234-241, Addison-Wesley/ACM Press, 1997.

[15] Ishii, H., et. al, "PingPongPlus: Design of an AthleticTangible Interface for Computer-Supported Cooperative Play”, CHI '99, May 15-20, Pittsburgh, May 1999.
[16] Kreuger, W. and B. Froehlich, "The Responsive Workbench", IEEE Computer Graphics and Applications, Vol. 14, No. 3, May, 1994, pp. 12-15.

[17] Mogensen, P. and Trigg, R., “Artefacts as Triggers for Participatory Design", Proceedings of the 1992 Participatory Design Conference, p. 55-62, Boston: Computer Professionals for Social Responsibility. 1992.

[18] Schwabe D., and Stenius M., "The Web Planetarium and Other Applications in the Extended Virtual Environment EVE”, Spring Conference on Computer Graphics, Budmerice, May 3rd-6th 2000

[19] Schäfer, K; Brauer, V; Bruns, F. W. “A new Approach to Human-Computer Interaction; Synchronous Modeling in Real and Virtual Spaces", in Proceedings of the DIS (Designing Interactive Systems), Amsterdam, NL, 1997.

[20] B. Shneiderman, "Direct Manipulation: A Step Beyond Programming Languages," IEEE Computer, vol. 16, no. 8, pp. 57-69, 1983.

[21] Underkoffler J. and Ishii H., "Urp: A Luminous-Tangible Workbench for Urban Planning and Design", CHI '99, 386393, May 1999.

[22] Ullmer, B., Ishii, H. and Glass, D., "MediaBlocks: Physical Containers, Transports, and Controls for Online Media," Computer Graphics Proceedings (SIGGRAPH '98), July, 1998. 